\title{
Effects of inoculation with plant growth promoting rhizobacteria (PGPRs) on different growth parameters of cold area rice variety, Fakre malakand
}

\author{
Bashir Ahmed ${ }^{1 \star}$, Midrarullah ${ }^{1,2}$ and M. Sajjad Mirza ${ }^{2}$ \\ ${ }^{1}$ Pharma biotech Research Laboratory, Centre of Biotechnology and Microbiology, University of Peshawar, KPK, \\ Pakistan. \\ ${ }^{2}$ National Institute for Biotechnology and Genetic Engineering (NIBGE), P.O. Box 577, Jhang Road, Faisalabad, \\ Pakistan.
}

Accepted 29 March, 2013

\begin{abstract}
The present study was conducted to isolate and characterize plant growth promoting rhizobacteria (PGPR) from the rhizosphere of rice grown in cold climate at Swat and to assess their impact on plant growth when used as inoculants. A total of 18 bacterial strains were isolated from roots and rhizosphere of rice. With the exception of 3 strains, all isolates showed IAA production in pure culture. Three bacterial strains (Azospirillum brasilense R1, Azospirillum lipoferum RSWT1 and Pseudomonas Ky1) were used to inoculate rice at two experimental sites in Swat (Agriculture Research Institute (N) Mingora and Udigram). Plant growth promotion was observed in all inoculated treatments over noninoculated control which was evident from increase in number of tillers, straw and grain yields and total weight of the plants. A. brasilense R1 was more effective in plant growth promotion than other strains. At ARIN Mingora, Swat, inoculation of rice variety Fakre Malakand with $A$. brasilense R1 increased the straw weight by $16.6 \%$, grain weight by $22.7 \%$ over control. Inoculation with A. lipoferum RSWT1 and Pseudomonas Ky1 increased grain weight by 4.8 to $13.5 \%$ and 17.3 to $18.5 \%$, respectively. At Udigram, Swat, inoculation with $A$. brasilense R1 increased the straw weight by $14.2 \%$ and grain weight by $22 \%$ than the control.
\end{abstract}

Key words: Azospirillum, Pseudomonas, inoculation of rice, effects of inoculation.

\section{INTRODUCTION}

Rice (Oryza sativa L.) is an important Kharif crop of Pakistan, ranking second to wheat as a staple food. Rice is a monocotyledonous plant belonging to the genus Oryza L., sub family Oryzoideae, in the family Poaceae (Graminae). Rice is cultivated between $36^{\circ} \mathrm{ES}$ and $55^{\circ}$ EN and grows from sea level to an altitude of $2,500 \mathrm{M}$ or even higher. Rice has gradually moved to occupy a predominant position in the agriculture economy of Pakistan. In Khyber Pakhtunkhwa (KPK), rice cultivation stands next to wheat and maize and is characterized by being grown under two different agro-climate conditions, that is, the plain and the upper mountainous valleys. Most of the cultivated area (81\%) is situated in the cooler, high altitude area of Malakand, Hazara Division and adjacent tribal areas of KPK. In Swat during 2008 to 2009, rice was grown in an area approximately 7349 hectares (ARIMS, 2009).

The average rice yield in the country and particularly in KPK is far behind what can be obtained from the potential of the crop. The increase in the yield of rice will be largely 
dependent upon availability of essential nutrients like nitrogen and phosphorus in the soil which can be supplied as chemical fertilizers. This leads to environmental pollution and health hazards. The use of chemical fertilizers is also too costly, especially nitrogen, which is one of the common limiting factor of rice production (Ladha and Reddy, 1995). In Khyber Pakhtunkhwa (KPK), rice cultivation stands next to wheat and maize and is characterized by being grown under two different agro-climate conditions, that is, the plain and the upper mountainous valleys. Most of the cultivated area $(81 \%)$ is situated in the cooler, high altitude area of Malakand, Hazara Division and adjacent tribal areas of KPK. In Swat during 2008 to 2009, rice was grown in an area approximately 7349 hectares (ARIMS, 2009).

The average rice yield in the country and particularly in KPK is far behind what can be obtained from the potential of the crop. The increase in the yield of rice will be largely dependent upon availability of essential nutrients like nitrogen and phosphorus in the soil which can be supplied as chemical fertilizers. This leads to environmental pollution and health hazards. The use of chemical fertilizers is also too costly, especially nitrogen, which is one of the common limiting factor of rice production (Ladha and Reddy, 1995).

A substitute is the use of plant growth promoting rhizobacteria (PGPR) as bacterial inoculants for crops (Glick et al., 1999; Ryu et al., 2005). PGPR inoculation to rice may effectively increase the surface area of roots and root weight (Cakmakci et al., 2007). Omar et al. (1989) reported that inoculation of rice with Azospirillum brasilense increased yield to 15 to $20 \%$. Tariq et al. (2007) reported that the application of PGPR to rice results in an increase of about $23 \%$ in the total biomass and $65 \%$ in the grain yield.

The present study was conducted with the objectives of isolating PGPRs from the rhizosphere of rice roots and testing the selected bacterial inoculants for rice grown at Agriculture Research Institute (N) Mingora, Swat and Udigram Swat.

\section{MATERIALS AND METHODS}

\section{Isolation of bacteria from rice roots and rhizosphere}

Roots of rice (O. sativa L.) variety Fakre Malakand along with the rhizosphere soil were collected from the plants grown in the fields of Udigram and Agriculture Research Institute (ARI) Mingora, Swat. They were placed in polythene bags and brought to the laboratory. The non-rhizosphere soil was removed by gentle shaking, leaving behind the rhizosphere soil. The samples were stored at $4^{\circ} \mathrm{C}$ and used for further studies. One gram of roots along with adhering soil was ground well with the help of a pestle and mortar. Serial dilutions (10x) were made and $100 \mu \mathrm{L}$ aliquots from $10^{-3}$ to $10^{-5}$ dilutions were spread on LB plates (Maniatis et al., 1982). Semi solid NFM (Okon et al., 1977) was incubated with $100 \mu \mathrm{L}$ of these serial dilutions. The inoculated plates and NFM vials were incubated for 24 to $72 \mathrm{~h}$ at $30^{\circ} \mathrm{C}$. Morphologically different colonies appearing on the growth medium were selected for further purifications. Isolated colonies were streaked on fresh plates with LB medium to get single-cell colonies. Bacterial growth obtained in NFM medium was streaked on NFM agar plates and incubated at $30^{\circ} \mathrm{C}$ for 24 to $72 \mathrm{~h}$. Single colonies were further streaked on fresh plates to get pure colonies. Single colonies appearing on the agar plates were transferred to a drop of sterilized water on a microscopic glass slide and observed under the light microscope (Nikon Japan). The bacterial cultures obtained were grown at $30^{\circ} \mathrm{C}$ for $24 \mathrm{~h}$ and preserved in glycerol $(20 \%)$ at $-20^{\circ} \mathrm{C}$.

\section{Identification and characterization of bacterial isolates}

Morphological and physiological tests were conducted to characterized bacterial strains. These tests include pigment production on nutrient agar medium, cell morphology and motility and growth at $30^{\circ} \mathrm{C}$ on NFM. Light microscope was used to study the cultural characteristics of the purified bacterial strains. All the strains were grown on Luria-Bertani (LB) agar medium (Maniatis et al., 1982) and incubated at $30^{\circ} \mathrm{C}$ for 24 to $48 \mathrm{~h}$. The purified cultures were maintained on LB agar slants. The colonies of bacterial culture were observed for colour, shape, size and motility and tentatively identified.

\section{Raising of rice nursery and inoculation of rice in the field experiment}

A fertile piece of land that has easy access to the water channel and convenient drainage system was selected to raise the rice nursery. The land was prepared by ploughing with tractor three times. The field was then irrigated. After one week, eradication of the weeds was carried out through ploughing and planking. The water was left to remain in the field during this process. In order to retain equal amount of water in the seed beds, the field was puddled well and thoroughly harrowed. Seeds of the selected rice were cultivated on 21 May 2008 at Agriculture Research Institute North (ARIN) Mingora and at Udigram Swat.

The water was drained out daily at night time in the initial stages of growth. Afterward, water was retained 2 to $4 \mathrm{~cm}$ deep in order to suppress weeds. After 30 days, that is, on 20 June 2008, the nursery was transplanted to the fields. During transplantation, the water depth was kept at $2 \mathrm{~cm}$ in the field. Bacterial inoculums (50 $\mathrm{mL}$ ) of the selected three bacterial strains were added to $2 \mathrm{~L}$ water and roots of the nursery seedling were inoculated for $1 \mathrm{~h}$. After inoculation of the rice seedlings, the remaining bacterial suspension was distributed equally in their respective beds. The seedling was brought and distributed throughout the field in their respective beds in the form of small bundles. The right number of seedling was detached from the bundles and inserted in the soil not shallower than $1.5 \mathrm{~cm}$ and not deeper than $3 \mathrm{~cm}$. Two seedlings per hill at 20 $x 20 \mathrm{~cm}$ distance were planted. The missing hills were replaced about 10 days after transplantation. Randomized complete block design was used in the present study with four treatments and four replicates. The plot size was $3 \times 3 \mathrm{~m}$ at Agriculture Research Institute (N), Mingora and $3 \times 5 \mathrm{~m}$ at Udigram, Swat. The number of rows at ARIN, Swat was $14 \times 15$ with 210 plants per plot and at Udigram was $12 \times 22$ with 330 plants per plot. After one week of transplantation, recommended chemical fertilizers $\left(120 \mathrm{~kg} \mathrm{ha}^{-1} \mathrm{~N}\right.$, $60 \mathrm{~kg} \mathrm{ha}^{-1} \mathrm{P}$ and $40 \mathrm{~kg} \mathrm{ha}^{-1} \mathrm{~K}$ ) were applied.

To record the total grain weight and straw weight from each plot, the grains were separated from the straw and their fresh weight was recorded. In order to carry out dry weight study of grains and straw, $5 \mathrm{~kg}$ of grains were dried in oven at $55^{\circ} \mathrm{C}$ for three days. After drying the plant material, dry weight was measured and the difference between fresh weight and dry weight was calculated. Keeping in view the loss per $\mathrm{kg}$, the total dry grain weight of each treatment was calculated. The dry straw weight and total dry weight 
Fakre Malakand, Agriculture Research Institute Mingora Swat

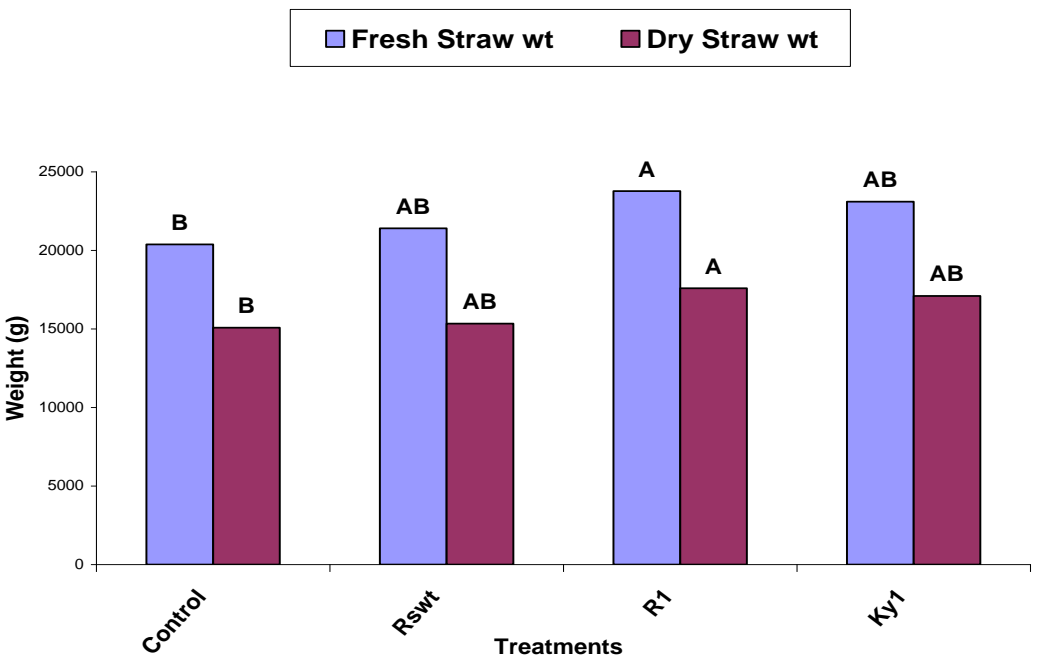

Figure 1. Effect of inoculated strains on straw weight of rice variety Fakre Malakand.

Fakre Malakand Agriculture Research Institute Mingora Swat

$\square$ Fresh Grain wt $\square$ Dry Grain wt

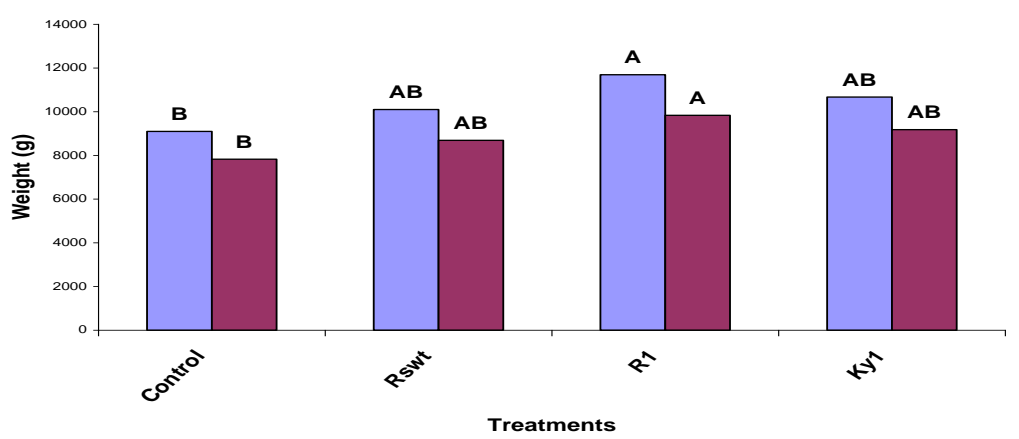

Figure 2. Effect of inoculated strains on straw weight of rice variety Fakre Malakand. Control: Non-inoculated; RSWT1: Azospirillum lipoferum; R1: Azospirillum brasilense Ky1: Pseudomonas. The values are an average of 4 replicates. Different letters given above the bars in the graphs show that value are different at $5 \%$ level of significance.

of the plants per plot and per treatment was also calculated following the same methodology. Statistical calculations were carried out by using MSTAT C program and LSD tests.

\section{RESULTS AND DISCUSSION}

A variety of bacteria are found in close association with rice. They have the potential to promote rice growth and can be used as biofertilizer (Meunchang et al., 2004).

Yield data collected from whole plots indicated that the inoculation of bacterial strains in both experimental fields resulted in more yields than non inoculated ones. The effect of the inoculated strains was positive on both fresh and dry weight. In the experiment conducted at Agricultural Research Institute (N) Mingora, Swat, inoculation of rice with $A$. brasilense R1 significantly increased the fresh and dry weight of straw, grain and total plant (straw + grain) weight. This increase was $16.6 \%$ in dry straw weight, $22.7 \%$ in dry grain weight and $19.8 \%$ in total plant dry weight over the non-inoculated control. The inoculation of rice with Pseudomonas Ky1 also showed significant increase in the straw weight by $13.4 \%$ total dry grain weight by $17.3 \%$ and total plant dry weight by $14.7 \%$ (Figures 1 to 3 ). Inoculation of 
Fakre Malakand, Agriculture Reseach Institute Mingora Swat

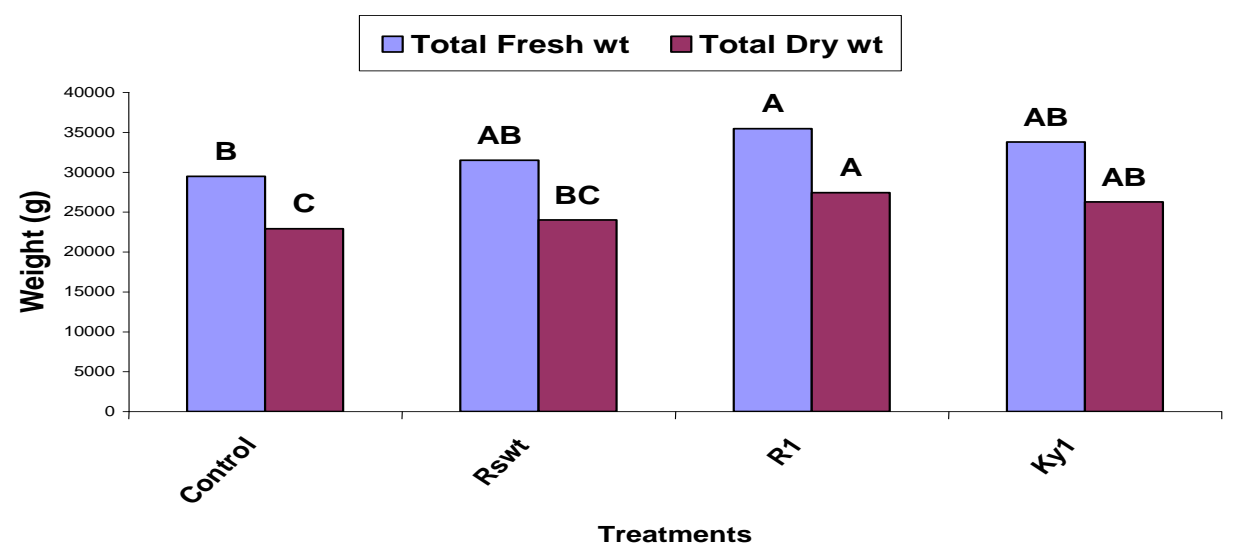

Figure 3. Effect of inoculated strains on total plant weight (straw +grain) of rice variety Fakre Malakand.

Fakre Malakand, Udigram Swat

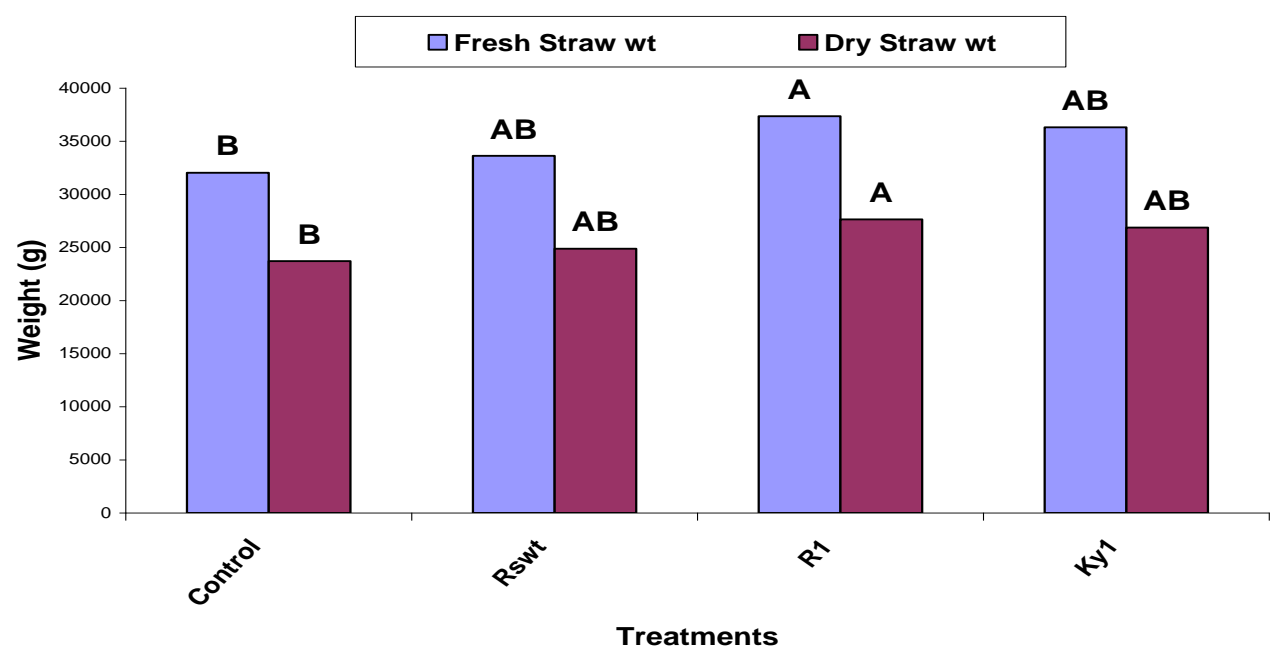

Figure 4. Effect of inoculated strains of straw weight of rice variety Fakre Malakand Control: Noninoculated; RSWT1: Azospirillum lipoferum; R1: Azospirillum brasilense Ky1: Pseudomonas. The values are an average of 4 replicates. Different letters given above the bars in the graphs show that value are different at $5 \%$ level of significance.

A. lipoferum RSWT1 showed no significant effect on the total plant dry matter; however an increase of $4.8 \%$ in the total dry grain weight of rice was shown. The results are parallel to the finding of Okon and Hadar (1987) who reported 10 to $30 \%$ increase in grain and forage field due to inoculation of Azospirillum to rice.

In the field experiment at Udigram, A. brasilense R1 was found to be the best inoculant as maximum increase in straw $(14.2 \%)$, grain $(22 \%)$ and total plant weight (17 $\%$ ) was obtained in plants inoculated with this strain (Figures 4 to 6).
Improvement in the growth of plants inoculated with two other bacterial strains ( $A$. lipoferum RSWT1 and Pseudomonas Ky1) treatments over non-inoculated control treatment was observed but it was not comparable to that obtained in treatment in which $A$. brasilense R1 was used as inoculant. The results were similar to those observed by Omar et al. (1989), who reported that inoculation of rice with $A$. brasilense increased yield by 15 to $20 \%$. This result is also in consistence with the findings of Tariq et al. (2007) who reported that the application of PGPR alleviated the 
Fakre Malakand Udigram

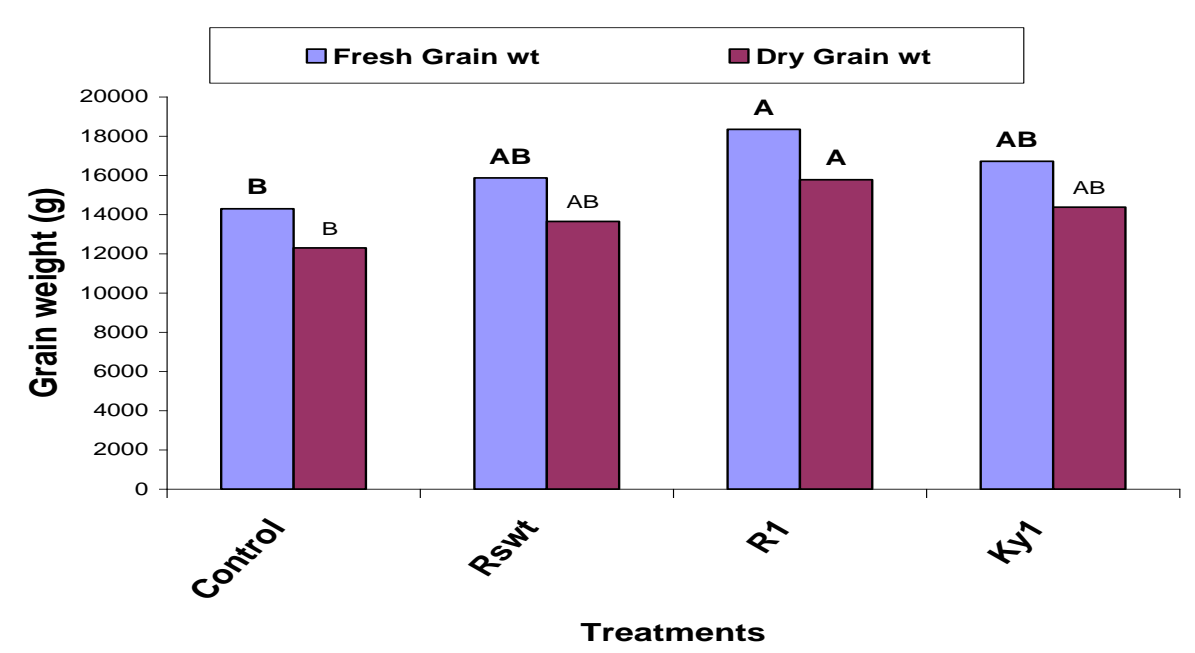

Figure 5. Effect of inoculated strains on grain weight of rice variety Fakre Malakand.

Fakre Malakand, Udigram Swat

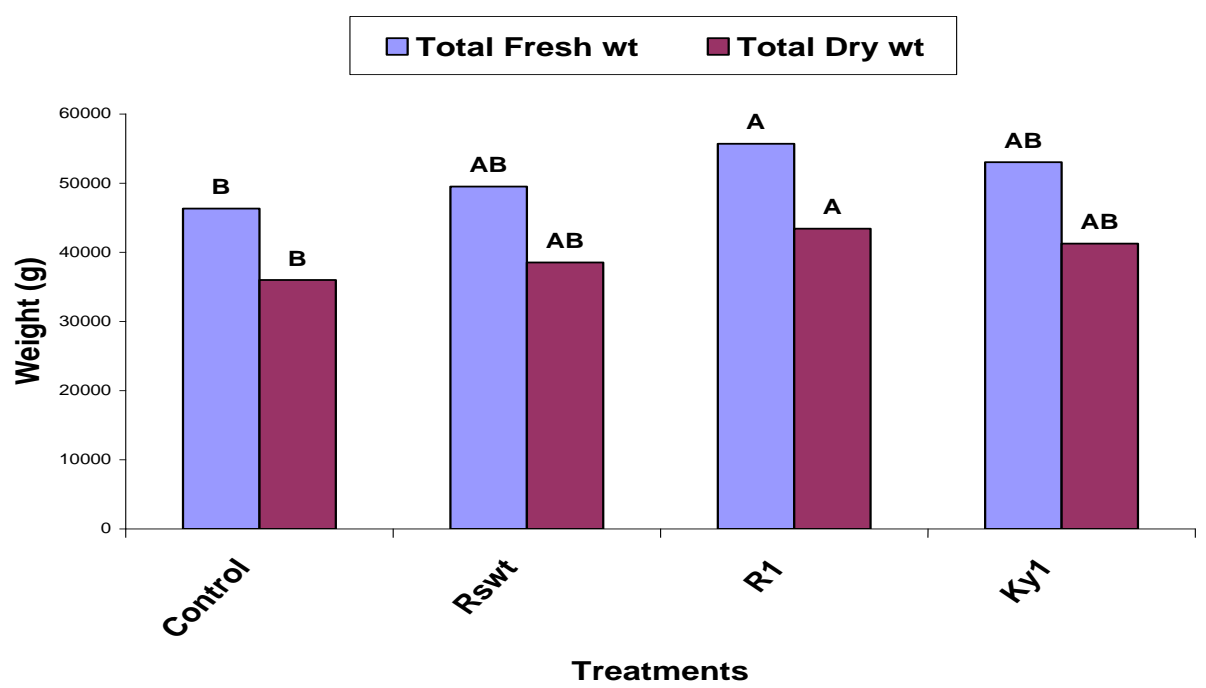

Figure 6. Effect of inoculated strains on total weight (straw + grain) of rice variety Fakre Malakand. Control: non-inoculated; RSWT1: Azospirillum lipoferum; R1: Azospirillum brasilense; Ky1: Pseudomonas. The values are an average of 4 replicates. Different letters given above the bars in the graphs show that value are different at $5 \%$ level of significance.

deficiency symptoms of $\mathrm{Zn}$ and increased $23 \%$ of the total biomass, $65 \%$ grain yield and also harvesting index and $\mathrm{Zn}$ concentration in the grains.

Comparative analysis of the single plant and whole plot data showed variation. In the single plant study at ARINS, Pseudomonas Ky1 showed more positive response on shoot weight and grain weight of rice than other inoculated strains. On the contrary, in the field experiments, $A$. brasilense $\mathrm{R} 1$ showed positive response on straw weight, grain weight and total weight than other treatments. In general, there was a good agreement between the results obtained from both experimental fields. Such variation between single plants and whole plot analysis proved that on the basis of single plant 
analysis or small scale cultivation, we cannot draw the exact picture about the effectiveness of a particular strain or comparing different inoculations.

This is the first study from Pakistan to demonstrate that PGPR can increase yield, growth and development of rice. In the present study, both the Azospirillum strains used as inoculants were isolated from rice grown in the same area. It has been reported than when locally isolated PGPR were used as inoculants, more crop yield than inoculation with type strain and control without inoculation was obtained (Fulchieri and Frioni, 1994).

Additional field studies in Swat area are required to confirm the beneficial role of bacterial inocula on growth and yield of variety Fakre Malakand and also locally isolated PGPR may be tested on other rice varieties grown in the area. This would help in developing a biofertilizer (inoculant) for use in agriculture in the future.

\section{REFERENCES}

Agriculture Research Institute Mingora Swat (2009). Statistical Division, Crop Reporting Services Swat, Pakistan.

Cakmakci R, Erat M, Erdogan UG, Donmez MF (2007). The influence of PGPR on growth parameters, antioxidant and pentose phosphate oxidative cycle enzymes in wheat and spinach plants. J. Plant Nutr. Soil Sci. 170:288-295.

Fulchieri M, Frioni L (1994). Azospirillum inoculation on maize (Zea mays): effect on yield in a field experiment in central Argentina. Soil Biol. Biochem. 26:921-923.
Glick BR, Patten CL, Holguin G, Penrose DM (1999). Biochemical and genetic mechanisms used by plant growth promoting bacteria. Imperial College Press, London, United Kingdom. p. 267.

Ladha JK, Reddy MM (1995). Extension of nitrogen fixation to rice: Necessity and possibilities. Geo. J. 35:363-372.

Maniatis T, Fritsch EF, Sambrook J (1982). Molecular cloning: $A$ laboratory manual. Cold Spring Harbor Laboratory, U.S.A. p. 545.

Meunchang S, Janzen HH, Rovira AD (2004). Phylogenetic and physiological characterization of indigenous Azospirillum isolates in Thailand. Soil Sci. Plant Nutr. 50: 413-421.

Okon Y, Albercht SL, Burris RH (1977). Method for growing Spirillum lipferum and for counting the pour cultures and in association with plant. Appl. Environ. Microbiol. 33:85-88.

Okon Y, Hadar Y (1987). Microbial inoculants as crop yield enhancers. CRC Crit. Rev. Biotechnol. 6: 61-79.

Omar N, Th Heulin P, Weinhard, Alaa El-Din MN (1989). Field inoculation of rice with in vitro selected plant growth promotingrhizobacteria. Agronomie 9:803-808.

Ryu CM, Hu CH, Locy RD, Kloepper JW (2005). Study of mechanisms for plant growth promotion elicited by rhizobacteria in Arabidopsis thaliana. Plant Soil 268:285-292.

Tariq M, Hameed S, Malik KA, Hafeez FY (2007). Plant root associated bacteria for Zinc mobilization in rice. Pak. J. Bot. 93:245-253. 